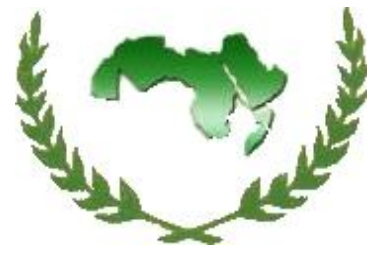

Arab Univ.

J. Agric. Sci.,

Ain Shams Univ.,

Cairo, 15(1), 141-155, 2007

\title{
EFFECT OF POLYETHYLENE GLYCOL (PEG) AND PLANTING METHODS ON SWEET CORN PRODUCTIVITY
}

\section{Abou El-Yazied, A. ${ }^{1}$; I.I. El-Oksh ${ }^{1}$; Rawia, E.I. El-Bassiouny ${ }^{2}$ and Manal M. Attia ${ }^{2}$}

1. Horticulture Department, Faculty of Agriculture, Ain Shams University, Shoubra El-Kheima, Cairo, Egypt

2. Phostharvest and Handling of Vegetable Department, Horticultural Research Institute, Agricultural Research Center, Giza, Egypt

Keywords: Sweet corn; Zea mays, L. var. Saccharata; seed Priming; PEG; Planting methods.

\begin{abstract}
This study was conducted in loamy soil at Qaha Experimental Station, Horticulture Research Institute, ARC, Kalubia governorate, during 2002/2003 and 2003/2004 seasons to study the effect of PEG and planting methods on sweet corn productivity. The obtained results indicated that GSS $9377 F_{1}$ hybrid plants produced vigorous growth and higher early and total yield than those of Endeavor $F_{1}$ hybrid. Direct seeding in the open field or under tunnels increased plant height, stem diameter, number of leaves/plant, plant fresh weight, unhusked ear weight, husked ear weight and total yield compared to transplanting methods. Transplanting produced the higher early yield either with open field or under tunnels. Primed seeds of sweet corn $\mathrm{F}_{1}$ hybrids (GSS 9377 and Endeavor) in PEG 6000 dalton for 6 hours at the concentration of $400 \mathrm{~g} / \mathrm{L}$ produced taller plants with thicker stem diameter, higher number of leaves/plant and greater fresh weight, higher unhusked and husked ear weight, ear length, higher TSS as well as early and total yield compared to check untreated seeds
\end{abstract}

\section{INTRODUCTION}

Fresh sweet corn (Zea mays, L. var. Saccharata) is a recently promising vegetable crop grown in Egypt. As an annual crop, the time from planting to seedling emergence or transplant establish- ment is a crucial phase in the production cycle. The uniformity and percentage of seedlings emergence of the direct seeding or percent of transplant stand can have major impacts on the final yield and quality, particularly when faced with physical stresses such as extreme temperatures. Bradford (1986) listed the results of osmotic seed priming on several vegetable crops and mentioned that there were generally positive results for many crop species indicated that there is potential for using seed priming to improve emergence under field conditions. It was added that the obstacle for the commercial application of seed priming is the variability of results among species, varieties and even seed lot. Waters et al. (1990) stated that use of transplants generally had little impact beyond decreasing percent of survival and plant height compared to direct-seeding. Whereas, Wyatt and Akridge (1993) reported that transplanting brought forward the harvesting date by 12-20 days compared with direct sowing. In general, direct sown sweet corn produced larger plants, higher yield and larger ears than transplanted sweet corn. Vik and Susort (1990), on sweet corn, mentioned that polyethylene tunnels reduced time for sowing or transplanting to harvest and this was the most important factor in increasing yield and plant quality. On average, tunnels increased yield by $29.4 \%$, increased the first grade yield by $28 \%$ and decreased the growing season by 16 days. Welbaum et al (2001) worked on methods of transplanting comparing with direct-seeded production, found that the direct-seeded plants were taller with better root development than those of transplants, in all years. Transplants produced smaller, lower-quality ears than did direct-seeded plants. 
This study was, therefore, performed to investigate the influences of seed priming, growing under low tunnel and transplanting on the early production of sweet corn.

\section{MATERIAL AND METHODS}

This study was conducted at Qaha Experimental Station, Vegetables Research Department, Ministry of Agriculture, during 2002/2003 and 2003/2004 seasons in loam soil. Sweet corn seeds of Endeavor $\mathrm{F}_{1}$ hybrid $\left(\mathrm{sh}_{2}\right)$ (obtained from Asgrow co. (USA)) and GSS9377 $\mathrm{F}_{1}$ hybrid $\left(\mathrm{sh}_{2}\right)$ (obtained from Syngenta co., Holland) were used in this experiment.

A preliminary experiment was conducted to find out the optimum method for seed priming under the experimental conditions as described by Bradford (1986). The obtained results indicated that PEG 6000 dalton at $400 \mathrm{~g} / \mathrm{L}$ and soaking for 6 hr was the best treatment and it was chosen for further experiment.

The objective of this experiment was to determine the performance of the most favorable priming treatment of sweet corn seeds at early planting date, i.e., low temperature conditions. Besides to examine the influence of some planting methods on seed performances, plant growth, earliness, yield and quality of ears under such field conditions.

Sweet corn seeds of both hybrids were primed at $400 \mathrm{~g} / \mathrm{L}$ PEG 6000 dalton for 6 hours and rinsed with distilled water five times till the traces of PEG were disappeared. On February 8 of 2003 and 2004, moist seeds of primed or check (unprimed) treatments were then sown in dry soil in hills, 4 seeds per hill, spaced $25 \mathrm{~cm}$ apart on one side of ridge of $70 \mathrm{~cm}$ apart. Irrigation was applied immediately after sowing; after emergence, the germinated seeds were thinned leaving two per hill.

As for transplanting, primed and check untreated sweet corn seeds were sown on the same day of direct seed treatments (February 8) in trays contained a mixture of peat moss and vermiculite $(1: 1 \mathrm{v} / \mathrm{v})$. The mixture was enriched with macro and microelements and treated with Benlete (1 $\mathrm{g} / \mathrm{L}$ ) according to the recommendations of the Egyptian Ministry of Agriculture "the recommended transplant production media for protected cultivation. The trays were kept under a plastic house for 15 days before replanting. Plants were hardened by subjecting them for 3 days to the open field conditions. In 2003 and 2004 seasons, the eighteen-day old plants were set up in the field at $25 \mathrm{~cm}$ apart on ridges of $70 \mathrm{~cm}$ width.

Half plots of the direct seed and transplant treatments were covered with transparent polyethylene tunnels (60 micron thickness) on the same day of sowing (February 8) or transplanting (February 26), the polyethylene tunnels were removed on March 18. The other half of plots of direct sowing and transplanting treatments were not covered with polyethylene, i.e., grown under the field conditions. The other agricultural management conducted according to the recommendation of Egyptian Ministry of Agriculture.

\section{The experimental design}

The experiment was factorial with three factors distributed in randomized complete block design with four replicates. The studied factors were hybrids (Endeavor $\mathrm{F}_{1}$ and GSS9377 $\mathrm{F}_{1}$ ), seed priming (primed and check), and planting methods (direct seed and transplanting) in the open field or under tunnels. The plot area was $12.25 \mathrm{~m}^{2}$, which consisted of 5 rows $70 \mathrm{~cm}$ apart and $3.5 \mathrm{~m}$ length.

\section{Data recorded}

Fifteen plants were randomly taken at harvest from each plot and number of leaves/plant, plant height $(\mathrm{cm})$ from the stalk base to tassel top, stem diameter $(\mathrm{cm})$ at the fourth node and plant fresh weight were recorded:

At harvest stage "in the milky stage", thirty ears from each plot were taken randomly to record ear length (ear base to top), husked and unhusked ear weight and TSS measured by a hand refractometer. Starch, total, reducing and non reducing sugars were measured colormetrically using spectrophotometer Model $6305 \mathrm{UV} /$ visible range with $520 \mathrm{~mm}$ wavelength according to Somogyi (1952) and Nelson (1974). Dry matter percent was determined by weighting one hundred gram of fresh kernels and then dried at $70^{\circ} \mathrm{C}$ until a constant weight. Early and total yield were also recorded as $\mathrm{kg} /$ feddan.

Statistical analysis was performed according to Snedecor and Cochran (1989).

\section{RESULT AND DISCUSSIONS}

\section{Vegetative growth}

Plants of GSS $9377 \mathrm{~F}_{1}$ hybrid were taller than those of Endeavor $F_{1}$ hybrid in both seasons, but the difference was not significant in the second 
season (Table, 1). Direct seeding either in the open field or under tunnel resulted in increasing the plant height compared to transplanting either in the open field or under tunnels in both seasons. No statistical differences were noticed between growing the plants either in the open field or under tunnel, regardless of using direct seeding or transplanting methods. These results were true in both seasons. This result agrees with that of Wyatt \& Mullins (1989) and with Wyatt and Akridge (1993).

Regarding the effect of seed priming, results indicated that the application of PEG gave the taller plants in both seasons compared to check treatment. Concerning the effect of the tested interactions, the interaction between cultivar and planting methods showed that GSS $9377 \mathrm{~F}_{1}$ hybrid which was direct seeded in the open field gave the tallest plants in 2003 season, but Endeavor $F_{1}$ hybrid which was direct seeded under tunnel gave the tallest plants in 2004 season. The rest of interactions were not significant.

As for stem diameter, plants of GSS $9377 \mathrm{~F}_{1}$ hybrid had higher values of stem diameter than those of Endeavor $F_{1}$ hybrid in both growing seasons (Table, 1).

Direct seeding either in open field or under tunnel gave thicker stems compared to transplanting in the open field or under tunnels, in both seasons. No significant differences were detected neither between the two direct seeding treatments nor the two transplanting ones.

Regarding the seed priming, seeds treated with PEG gave plants with the higher values of stem diameter compared to check treatment.

These results are in agreement with those of Bennett (1986), who stated that hydration increased the size of plants cut at 2 to 4 leaf stage. The interaction between cultivar and planting method indicated that plants of GSS $9377 \mathrm{~F}_{1}$ hybrid planted with direct seed in the open field had the highest values of stem diameter in both seasons compared to any other interaction treatment. No significant differences were noticed for the rest of interactions.

With respect to number of leaves, plants of GSS $9377 F_{1}$ hybrid produced higher number of leaves than those of Endeavor $F_{1}$ hybrid in both seasons (Table, 2). Direct seeded plants grown in the open field or under tunnel significantly gave higher number of leaves than those transplanted in the open field or tunnel treatments.

Concerning seed priming, seeds treated with PEG produced plants with high number of leaves compared to those of check treatment in the two growing seasons. All tested interactions were not statistically significant except the interaction between cultivar and planting method in the second season where Endeavor $F_{1}$ hybrid with direct seeding under tunnel gave plants with the highest number of leaves.

As to fresh weight, plants of GSS $9377 \mathrm{~F}_{1}$ hybrid had higher fresh weight compared with those of Endeavor $F_{1}$ hybrid in both studied seasons (Table, 2). Direct seeding either in the open field or under tunnels was more favourable for the simulation of fresh weight production compared to transplanting either in the open field or under tunnels. No significant differences were observed between the two transplanting methods in both seasons, but the direct seed in the open field significantly increased the plant fresh weight compared to direct seed under tunnels, only in the first season. Regarding the effect of seed priming with polyethylene glycol (PEG), the plant fresh weight increased by seed priming compared to check (dry seed) treatment in both seasons. This result agrees with that of Bennett and Waters (1987b), who stated that the plant size of sweet corn was increased by using hydration treatments at an early ( 2 to 4 leaf) vegetative stage.

The interaction among the tested factors were not significant except for the interaction between cultivar and planting method, where the obtained data showed that GSS $9377 \mathrm{~F}_{1}$ hybrid with direct seeding in the open field was the best treatment for increasing the fresh weight of sweet corn plants whereas transplanting the Endeavor in the open field gave the lowest values. Generally, direct seeding method increased plant fresh weight compared to transplanting method. Similar results were reported by Aguyoh et al (1999).

It could be concluded that GSS $9377 \mathrm{~F}_{1}$ hybrid produced plants with vigorous growth, expressed as plant height, stem diameter, leaf number and fresh weight, more than those of Endeavor $F_{1}$ hybrid. This might be attributed the genetical differences between the two hybrids.

Seed priming with PEG resulted in higher vegetative growth compared to the check treatment. This may be due to improving the seedling growth at early stage of plant development under cold weather which may enhance the final plant growth.

Transplanting reduced the plant height and all vegetative growth parameters and this may be attributed to the restricting of root growth and development in the root cell trays compared to direct 
Table 1. Effect of hybrid, planting method and seed priming on plant height and stem diameter of sweet corn $F_{1}$ hybrids in 2002/2003 and 2003/2004 seasons.

\begin{tabular}{|c|c|c|c|c|c|c|c|}
\hline \multirow{2}{*}{\multicolumn{2}{|c|}{ Planting method }} & \multicolumn{3}{|c|}{$2002 / 2003$} & \multicolumn{3}{|c|}{$2003 / 2004$} \\
\hline & & GSS & Endeavor & Mean & GSS & Endeavor & Mean \\
\hline \multirow{4}{*}{ D. seed open } & & \multicolumn{6}{|c|}{ Plant height (cm) } \\
\hline & Priming & 175.24 & 147.94 & 161.59 & 145.63 & 139.12 & 142.37 \\
\hline & Check & 167.32 & 137.38 & 152.35 & 142.01 & 135.38 & 138.69 \\
\hline & Mean & 171.28 & 142.66 & 156.97 & 143.82 & 137.25 & 140.53 \\
\hline \multirow[t]{3}{*}{ D. seed Tunnel } & Priming & 161.19 & 149.14 & 155.16 & 143.88 & 169.73 & 156.80 \\
\hline & Check & 158.68 & 144.26 & 151.47 & 134.03 & 143.99 & 139.01 \\
\hline & Mean & 159.93 & 146.70 & 153.31 & 138.95 & 156.86 & 147.90 \\
\hline \multirow[t]{3}{*}{ Transplanting open } & Priming & 159.81 & 139.91 & 149.86 & 136.66 & 117.73 & 127.19 \\
\hline & Check & 149.82 & 126.26 & 138.04 & 131.45 & 116.59 & 124.02 \\
\hline & Mean & 154.81 & 133.08 & 143.94 & 134.05 & 117.16 & 125.60 \\
\hline \multirow{3}{*}{$\begin{array}{c}\text { Transplanting tun- } \\
\text { nel }\end{array}$} & Priming & 154.27 & 141.21 & 147.74 & 131.73 & 125.29 & 128.51 \\
\hline & Check & 147.26 & 130.84 & 139.05 & 127.43 & 114.21 & 120.82 \\
\hline & Mean & 150.76 & 136.02 & 143.39 & 129.58 & 119.75 & 124.66 \\
\hline \multirow[t]{3}{*}{ Mean } & Priming & 162.63 & 144.55 & 153.59 & 139.47 & 137.97 & 138.72 \\
\hline & Check & 155.77 & 134.68 & 145.22 & 133.73 & 127.54 & 130.63 \\
\hline & Mean & 159.19 & 139.62 & & 136.60 & 132.75 & \\
\hline \multicolumn{2}{|c|}{ LSD at 5\% level for Hybrid (H) } & & 4.34 & & & NS & \\
\hline \multicolumn{2}{|c|}{ Planting method (PM) } & & 6.14 & & & 8.34 & \\
\hline \multicolumn{2}{|l|}{ Seed priming (SP) } & & 4.34 & & & 5.89 & \\
\hline \multicolumn{2}{|l|}{$\mathrm{H} \times \mathrm{PM}$} & & 8.67 & & & 11.77 & \\
\hline \multicolumn{2}{|l|}{$\mathrm{H} \times \mathrm{SP}$} & & NS & & & NS & \\
\hline \multicolumn{2}{|l|}{$\mathrm{PM} \times \mathrm{SP}$} & & NS & & & NS & \\
\hline \multicolumn{2}{|l|}{$\mathrm{H} \times \mathrm{PM} . \times \mathrm{SP}$} & & NS & & & NS & \\
\hline \multirow{4}{*}{ D. seed open } & & \multicolumn{6}{|c|}{ Stem diameter $(\mathrm{mm})$} \\
\hline & Priming & 20.44 & 17.95 & 19.19 & 20.63 & 17.20 & 18.91 \\
\hline & Check & 18.67 & 17.34 & 18.01 & 18.39 & 16.95 & 17.67 \\
\hline & Mean & 19.55 & 17.64 & 18.59 & 19.51 & 17.07 & 18.29 \\
\hline \multirow[t]{3}{*}{ D. seed Tunnel } & Priming & 18.82 & 19.04 & 18.93 & 19.39 & 17.87 & 18.63 \\
\hline & Check & 17.16 & 17.68 & 17.42 & 17.33 & 17.36 & 17.34 \\
\hline & Mean & 17.99 & 18.36 & 18.17 & 18.36 & 17.61 & 17.98 \\
\hline \multirow[t]{3}{*}{ Transplanting open } & Priming & 18.71 & 15.19 & 16.95 & 17.41 & 15.31 & 16.36 \\
\hline & Check & 17.30 & 14.98 & 16.14 & 15.95 & 14.70 & 15.32 \\
\hline & Mean & 18.01 & 15.08 & 16.54 & 16.68 & 15.01 & 15.84 \\
\hline \multirow{3}{*}{$\begin{array}{c}\text { Transplanting } \\
\text { tunnel }\end{array}$} & Priming & 18.44 & 15.76 & 17.10 & 16.69 & 16.25 & 16.46 \\
\hline & Check & 17.16 & 15.15 & 16.15 & 15.31 & 13.78 & 14.54 \\
\hline & Mean & 17.80 & 15.45 & 16.62 & 16.00 & 15.01 & 15.50 \\
\hline Mean & Priming & 19.10 & 16.98 & 18.04 & 18.53 & 16.66 & 17.59 \\
\hline & Check & 17.57 & 16.29 & 16.93 & 16.74 & 15.69 & 16.21 \\
\hline & Mean & 18.34 & 16.64 & & 17.64 & 16.18 & \\
\hline LSD at $5 \%$ level & or Hybrid (1 & & 0.57 & & 0.45 & & \\
\hline Planting method & PM) & & 0.81 & & 0.64 & & \\
\hline Seed priming (SI & & & 0.57 & & 0.45 & & \\
\hline $\mathrm{H} \times \mathrm{PM}$ & & & 1.14 & & 1.14 & & \\
\hline $\mathrm{H} \times \mathrm{SP}$ & & & NS & & NS & & \\
\hline $\mathrm{PM} \times \mathrm{SP}$ & & & NS & & NS & & \\
\hline $\mathrm{H} \times \mathrm{PM} . \times \mathrm{SP}$ & & & NS & & NS & & \\
\hline
\end{tabular}


Table 2. Effect of hybrid, planting method and seed priming on number of leaves/plant and plant fresh weight of sweet corn $F_{1}$ hybrids in 2002/2003 and 2003/2004 seasons.

\begin{tabular}{|c|c|c|c|c|c|c|c|}
\hline \multirow{2}{*}{\multicolumn{2}{|c|}{ Planting method }} & \multicolumn{3}{|c|}{$2002 / 2003$} & \multicolumn{3}{|c|}{$2003 / 2004$} \\
\hline & & GSS & Endeavor & Mean & GSS & Endeavor & Mean \\
\hline \multirow{3}{*}{ D. seed open } & & \multicolumn{6}{|c|}{ Number of leaves per plant } \\
\hline & Priming & 10.86 & 9.88 & 10.37 & 9.50 & 9.09 & 9.29 \\
\hline & Check & 10.49 & 9.49 & 9.99 & 9.01 & 8.66 & 8.83 \\
\hline \multirow{3}{*}{ D. seed Tunnel } & Mean & 10.67 & 9.69 & 10.18 & 9.25 & 8.87 & 9.06 \\
\hline & Priming & 10.59 & 10.20 & 10.39 & 9.23 & 9.96 & 9.59 \\
\hline & Check & 10.10 & 9.59 & 9.84 & 8.68 & 9.35 & 9.01 \\
\hline \multirow{4}{*}{ Transplanting open } & Mean & 10.34 & 9.89 & 10.11 & 8.95 & 9.65 & 9.30 \\
\hline & Priming & 10.21 & 8.97 & 9.59 & 8.48 & 6.73 & 7.60 \\
\hline & Check & 9.79 & 8.51 & 9.15 & 7.97 & 6.17 & 7.07 \\
\hline & Mean & 10.00 & 8.74 & 9.37 & 8.22 & 6.45 & 7.33 \\
\hline \multirow[t]{3}{*}{ Transplanting tunnel } & Priming & 9.62 & 9.10 & 9.36 & 8.04 & 7.35 & 7.69 \\
\hline & Check & 9.45 & 8.03 & 8.74 & 7.42 & 6.30 & 6.86 \\
\hline & Mean & 9.53 & 8.56 & 9.05 & 7.73 & 6.82 & 7.27 \\
\hline \multirow[t]{3}{*}{ Mean } & Priming & 10.32 & 9.54 & 9.93 & 8.81 & 8.28 & 8.54 \\
\hline & Check & 9.96 & 8.90 & 9.43 & 8.27 & 7.62 & 7.94 \\
\hline & Mean & 10.14 & 9.22 & & 8.54 & 7.95 & \\
\hline \multicolumn{2}{|c|}{ LSD at 5\% level for Hybrid (H) } & & 0.34 & & & 0.15 & \\
\hline \multicolumn{2}{|l|}{ Planting method (PM) } & & 0.48 & & & 0.22 & \\
\hline \multicolumn{2}{|l|}{ Seed priming (SP) } & & 0.34 & & & 0.15 & \\
\hline \multicolumn{2}{|l|}{$\mathrm{H} \times \mathrm{PM}$} & & NS & & & 0.31 & \\
\hline \multicolumn{2}{|l|}{$\mathrm{H} \times \mathrm{SP}$} & & NS & & & NS & \\
\hline \multicolumn{2}{|l|}{$\mathrm{PM} \times \mathrm{SP}$} & & NS & & & NS & \\
\hline \multicolumn{2}{|l|}{$\mathrm{H} \times \mathrm{PM} . \times \mathrm{SP}$} & & NS & & & NS & \\
\hline \multirow{4}{*}{ D. seed open } & & \multicolumn{6}{|c|}{ Plant fresh weight (g) } \\
\hline & Priming & 319.85 & 270.12 & 294.98 & 372.91 & 282.36 & 327.63 \\
\hline & Check & 301.37 & 176.65 & 239.01 & 296.00 & 245.51 & 270.75 \\
\hline & Mean & 310.61 & 223.38 & 266.99 & 334.45 & 263.93 & 299.19 \\
\hline \multirow[t]{3}{*}{ D. seed Tunnel } & Priming & 245.26 & 279.89 & 262.57 & 323.77 & 339.04 & 331.40 \\
\hline & Check & 216.70 & 236.13 & 226.41 & 259.04 & 292.82 & 275.93 \\
\hline & Mean & 230.98 & 258.01 & 244.49 & 291.40 & 315.93 & 303.66 \\
\hline \multirow[t]{3}{*}{ Transplanting open } & Priming & 279.18 & 191.17 & 235.17 & 215.03 & 180.43 & 197.73 \\
\hline & Check & 220.91 & 146.63 & 183.77 & 179.48 & 151.79 & 165.63 \\
\hline & Mean & 250.04 & 168.90 & 209.47 & 197.25 & 166.11 & 181.68 \\
\hline \multirow[t]{3}{*}{ Transplanting tunnel } & Priming & 239.09 & 208.39 & 223.74 & 210.28 & 199.20 & 204.74 \\
\hline & Check & 188.55 & 152.11 & 170.33 & 162.63 & 160.14 & 161.38 \\
\hline & Mean & 213.82 & 180.25 & 197.03 & 186.45 & 179.67 & 183.06 \\
\hline Mean & Priming & 270.84 & 237.39 & 254.11 & 280.49 & 250.26 & 265.37 \\
\hline & Check & 231.38 & 177.88 & 204.63 & 224.29 & 212.56 & 218.42 \\
\hline & Mean & 251.36 & 207.64 & & 252.39 & 231.41 & \\
\hline LSD at 5\% level for $\mathrm{Hyb}$ & $\mathrm{d}(\mathrm{H})$ & & 11.95 & & & 15.77 & \\
\hline Planting method (PM) & & & 16.89 & & & 22.30 & \\
\hline Seed priming (SP) & & & 11.95 & & & 15.17 & \\
\hline $\mathrm{H} \times \mathrm{PM}$ & & & 23.84 & & & 31.48 & \\
\hline $\mathrm{H} \times \mathrm{SP}$ & & & NS & & & NS & \\
\hline $\mathrm{PM} \times \mathrm{SP}$ & & & NS & & & NS & \\
\hline $\mathrm{H} \times \mathrm{PM} . \times \mathrm{SP}$ & & & NS & & & NS & \\
\hline
\end{tabular}


seeding which permits more root extension and development. Therefore, the plant growth was enhanced with direct seeding compared to transplanting. Similar results were reported by Bennett (1986); Bennett \& Waters (1987a \& b); Wyatt \& Mullins (1989); Wyatt \& Akridge (1993) and Aguyah et al (1999).

\section{Ear quality}

The unhusked ear weight of GSS $9377 \mathrm{~F}_{1}$ hybrid was heavier compared to that of Endeavor in both seasons (Table, 3). Direct seeding in the open field gave the heaviest ears compared to the other methods in both seasons. However, no significant difference was observed between direct seeding in the open field and under tunnel in the first season, this may be attributed to the recovery time needed in replanting processes. Similar results were reported by Wyatt \& Akridge (1993) and Welbaum et al (2001), who found that direct seeding produced larger ears compared to transplanting method.

Seed priming enhanced the unhusked ear weight compared to check treatment in both seasons. As for the studied interactions, the hybrid combined with planting method was significant. The heaviest ears were produced from GSS 9377 $\mathrm{F}_{1}$ hybrid when direct seeded in the open field compared to the remainder treatments, in both seasons. No significant differences were noticed in both seasons between cultivar, planting method and seed priming. However, the heaviest unhusked ear weight was insignificantly recorded for GSS $9377 F_{1}$ hybrid when seeds were primed with PEG and planted directly in the open field.

Respecting the husked ear weight, plant of GSS $9377 F_{1}$ hybrid produced heavier husked ear weight compared to that of Endeavor $F_{1}$ in both seasons. Plants produced by direct seeding in the open field gave the heaviest husked ear compared to the other treatments. However, the difference was not significant in first season. The husked ears obtained from seed-primed plants were heavier than those of check treatment, although the difference was insignificant in the second season.

As for the interactions, all tested interactions were insignificant in both seasons, except for the interaction between hybrid and planting method in the first season, ears of GSS $9377 \mathrm{~F}_{1}$ hybrid were the heaviest when primed seeds were sown in the open field.

As for ear length, the ears of GSS $9377 \mathrm{~F}_{1}$ hybrid were taller than those of Endeavor $F_{1}$ hybrid in both seasons (Table, 4). No significant differ- ences in ear length were noticed among the investigated planting methods in both seasons. Treating the seeds with PEG resulted in higher ear length compared to check treatment; however, the difference was insignificant in the first season.

As to the interactions, the only significant one was between hybrid and planting method. The highest values of ear length were recorded for those of GSS $3977 F_{1}$ hybrid when seeds were directly sown in the open field compared to the other treatments. This held true in both seasons. It could be concluded from the previously mentioned results on ear characters that using the GSS 9377 $\mathrm{F}_{1}$ hybrid, direct seeding in the open field or seed priming significantly improved the weight of husked and husked ear. Seed priming improved also ear length and diameter.

\section{Chemical characters of kernels}

\section{Non reducing sugar (sucrose)}

Data presented in Table (5) showed that no significant difference between hybrids was found in the first season but GSS $9377 \mathrm{~F}_{1}$ hybrid recorded significantly higher sucrose percent compared to Endeavor $\mathrm{F}_{1}$ hybrid in the second season. Similarly no significant difference was noticed among the planting methods in 2003 season but in 2004 season direct seeding methods either in the open field or under tunnel significantly improved the non reducing sugar percent compared to the transplanting methods. Seeds primed with PEG produced plants having higher non reducing sugars in their kernels compared to check treatment in both seasons.

Concerning the interaction between hybrid and planting methods, GSS $9377 \mathrm{~F}_{1}$ hybrid with direct seeding under tunnel or Endeavor $F_{1}$ hybrid with direct seeding in the open field recorded the highest sucrose percent compared to other treatments. Seed priming with GSS $9377 \mathrm{~F}_{1}$ hybrid gave the highest significant values in sucrose percent in the second season. No significant differences were found with the interaction between planting methods and seed priming as well as among hybrid, planting method and seed priming, in both seasons.

\section{Reducing sugars}

Kernels of GSS $9377 \mathrm{~F}_{1}$ hybrid recorded significantly higher reducing sugars than those of Endeavor $F_{1}$ hybrid in both seasons. No significant 
Table 3. Effect of hybrid, planting method and seed priming on unhusked and husked ear weight of sweet corn $\mathrm{F}_{1}$ hybrids in 2002/2003 and 2003/2004 seasons.

\begin{tabular}{|c|c|c|c|c|c|c|c|}
\hline \multirow{2}{*}{\multicolumn{2}{|c|}{ Planting method }} & \multicolumn{3}{|c|}{$2002 / 2003$} & \multicolumn{3}{|c|}{$2003 / 2004$} \\
\hline & & GSS & Endeavor & Mean & GSS & Endeavor & Mean \\
\hline & & \multicolumn{6}{|c|}{ Unhusked ear weight (g) } \\
\hline \multirow{3}{*}{ D. seed open } & Priming & 319.44 & 255.87 & 287.65 & 337.79 & 239.27 & 288.53 \\
\hline & Check & 305.63 & 241.25 & 273.44 & 320.20 & 236.85 & 278.31 \\
\hline & Mean & 312.53 & 248.56 & 280.54 & 328.99 & 237.85 & 283.42 \\
\hline \multirow[t]{3}{*}{ D. seed Tunnel } & Priming & 274.47 & 273.33 & 273.90 & 281.38 & 262.41 & 271.89 \\
\hline & Check & 265.96 & 263.62 & 264.79 & 267.68 & 234.78 & 251.23 \\
\hline & Mean & 270.21 & 268.47 & 269.34 & 274.53 & 248.59 & 261.56 \\
\hline \multirow[t]{3}{*}{ Transplanting open } & Priming & 279.39 & 245.27 & 262.33 & 279.85 & 247.14 & 263.49 \\
\hline & Check & 261.07 & 235.59 & 248.33 & 255.76 & 233.35 & 244.55 \\
\hline & Mean & 270.23 & 240.43 & 255.33 & 267.80 & 240.24 & 254.02 \\
\hline \multirow[t]{3}{*}{ Transplanting tunnel } & Priming & 273.79 & 250.01 & 261.89 & 273.12 & 248.92 & 261.02 \\
\hline & Check & 270.93 & 244.24 & 257.58 & 261.88 & 223.52 & 242.70 \\
\hline & Mean & 272.36 & 247.12 & 259.74 & 267.50 & 236.22 & 251.86 \\
\hline \multirow[t]{3}{*}{ Mean } & Priming & 286.77 & 256.12 & 271.44 & 293.03 & 249.43 & 271.23 \\
\hline & Check & 275.89 & 246.17 & 261.03 & 276.38 & 232.02 & 254.20 \\
\hline & Mean & 281.33 & 251.15 & & 284.71 & 240.73 & \\
\hline \multicolumn{2}{|c|}{ LSD at 5\% level for Hybrid $(\mathrm{H})$} & & 9.90 & & 12.90 & & \\
\hline \multicolumn{2}{|l|}{ Planting method (PM) } & & 14.01 & & 18.24 & & \\
\hline \multicolumn{2}{|l|}{ Seed priming (SP) } & & 9.90 & & 12.90 & & \\
\hline \multicolumn{2}{|l|}{$\mathrm{H} \times \mathrm{PM}$} & & 19.76 & & 25.75 & & \\
\hline \multicolumn{2}{|l|}{$\mathrm{H} \times \mathrm{SP}$} & & NS & & NS & & \\
\hline \multicolumn{2}{|l|}{$\mathrm{PM} \times \mathrm{SP}$} & & NS & & NS & & \\
\hline \multicolumn{2}{|l|}{$\mathrm{H} \times$ PM. $\times \mathrm{SP}$} & & NS & & NS & & \\
\hline \multirow{4}{*}{ D. seed open } & & \multicolumn{6}{|c|}{ Husked ear weight (g) } \\
\hline & Priming & 228.79 & 184.84 & 206.81 & 213.43 & 174.27 & 193.85 \\
\hline & Check & 212.20 & 176.09 & 194.14 & 198.67 & 169.41 & 184.04 \\
\hline & Mean & 220.49 & 180.46 & 200.47 & 206.05 & 171.84 & 188.94 \\
\hline \multirow{3}{*}{ D. seed Tunnel } & Priming & 202.48 & 195.68 & 199.08 & 191.76 & 180.72 & 186.24 \\
\hline & Check & 196.07 & 194.91 & 195.49 & 181.77 & 163.77 & 172.77 \\
\hline & Mean & 199.27 & 195.29 & 193.05 & 186.76 & 172.24 & 179.50 \\
\hline \multirow[t]{3}{*}{ Transplanting open } & Priming & 207.06 & 179.05 & 193.05 & 188.73 & 172.98 & 180.85 \\
\hline & Check & 183.60 & 176.28 & 179.94 & 186.48 & 166.78 & 176.63 \\
\hline & Mean & 195.33 & 177.66 & 186.49 & 187.60 & 169.88 & 178.74 \\
\hline \multirow[t]{3}{*}{ Transplanting tunnel } & Priming & 203.61 & 182.46 & 193.90 & 183.78 & 179.04 & 181.41 \\
\hline & Check & 194.57 & 173.23 & 188.46 & 171.34 & 160.09 & 165.71 \\
\hline & Mean & 199.09 & 177.84 & 197.99 & 177.56 & 169.56 & 173.56 \\
\hline Mean & Priming & 210.48 & 185.51 & 188.37 & 194.42 & 176.75 & 185.58 \\
\hline & Check & 196.61 & 180.13 & & 184.56 & 165.01 & 174.78 \\
\hline & Mean & 203.55 & 182.82 & & 189.49 & 170.88 & \\
\hline LSD at $5 \%$ level for $\mathrm{Hyb}$ & $\mathrm{d}(\mathrm{H})$ & & 8.61 & & & 7.47 & \\
\hline Planting method (PM) & & & NS & & & 10.56 & \\
\hline Seed priming (SP) & & & 8.61 & & & NS & \\
\hline $\mathrm{H} \times \mathrm{PM}$ & & & 17.19 & & & NS & \\
\hline $\mathrm{H} \times \mathrm{SP}$ & & & NS & & & NS & \\
\hline $\mathrm{PM} \times \mathrm{SP}$ & & & NS & & & NS & \\
\hline $\mathrm{H} \times \mathrm{PM} . \times \mathrm{SP}$ & & & NS & & & NS & \\
\hline
\end{tabular}


Table 4. Effect of hybrid, planting method and seed priming on ear length of sweet corn $\mathrm{F}_{1}$ hybrids in 2002/2003 and 2003/2004 seasons.

\begin{tabular}{|c|c|c|c|c|c|c|c|}
\hline \multirow{2}{*}{\multicolumn{2}{|c|}{ Planting method }} & \multicolumn{3}{|c|}{$2002 / 2003$} & \multicolumn{3}{|c|}{$2003 / 2004$} \\
\hline & & GSS & Endeavor & Mean & GSS & Endeavor & Mean \\
\hline & & \multicolumn{6}{|c|}{ Ear length (cm) } \\
\hline \multirow[t]{3}{*}{ D. seed open } & Priming & 20.45 & 16.35 & 18.40 & 20.45 & 16.59 & 18.52 \\
\hline & Check & 19.84 & 16.18 & 18.01 & 19.65 & 16.26 & 17.95 \\
\hline & Mean & 20.14 & 16.26 & 18.20 & 20.05 & 16.42 & 18.23 \\
\hline \multirow[t]{3}{*}{ D. seed Tunnel } & Priming & 17.79 & 18.25 & 18.02 & 19.45 & 18.34 & 18.89 \\
\hline & Check & 17.23 & 18.09 & 17.66 & 18.59 & 17.14 & 17.86 \\
\hline & Mean & 17.51 & 18.17 & 17.84 & 19.02 & 17.74 & 18.38 \\
\hline \multirow[t]{3}{*}{ Transplanting open } & Priming & 20.46 & 16.29 & 18.37 & 20.12 & 17.24 & 18.68 \\
\hline & Check & 18.98 & 16.06 & 17.52 & 19.13 & 16.77 & 17.95 \\
\hline & Mean & 19.72 & 16.17 & 17.94 & 19.62 & 17.01 & 18.31 \\
\hline \multirow[t]{3}{*}{ Transplanting tunnel } & Priming & 17.61 & 17.23 & 17.42 & 19.45 & 17.44 & 18.44 \\
\hline & Check & 17.56 & 17.20 & 17.38 & 18.41 & 16.54 & 17.47 \\
\hline & Mean & 17.58 & 17.21 & 17.41 & 18.93 & 16.99 & 17.96 \\
\hline \multirow[t]{3}{*}{ Mean } & Priming & 19.08 & 17.03 & 18.05 & 19.87 & 17.40 & 18.63 \\
\hline & Check & 18.40 & 16.88 & 17.64 & 18.94 & 16.68 & 17.81 \\
\hline & Mean & 18.74 & 16.96 & & 19.41 & 17.04 & \\
\hline \multicolumn{3}{|c|}{ LSD at 5\% level for Hybrid (H) } & 0.44 & & 0.39 & & \\
\hline \multicolumn{3}{|c|}{ Planting method (PM) } & NS & & NS & & \\
\hline \multicolumn{2}{|c|}{ Seed priming (SP) } & & NS & & 0.39 & & \\
\hline \multicolumn{2}{|l|}{$\mathrm{H} \times \mathrm{PM}$} & & 0.87 & & 0.78 & & \\
\hline \multicolumn{2}{|l|}{$\mathrm{H} \times \mathrm{SP}$} & & NS & & NS & & \\
\hline \multicolumn{2}{|l|}{$\mathrm{PM} \times \mathrm{SP}$} & & NS & & NS & & \\
\hline \multicolumn{2}{|c|}{$\mathrm{H} \times \mathrm{PM} . \times \mathrm{SP}$} & & NS & & NS & & \\
\hline
\end{tabular}


Table 5. Effect of hybrid, planting method and seed priming on non-reducing and reducing sugars (g/100 $\mathrm{g}$ fresh weight) in kernels of sweet corn hybrids in 2002/2003 and 2003/2004 seasons.

\begin{tabular}{|c|c|c|c|c|c|c|c|}
\hline \multirow{2}{*}{\multicolumn{2}{|c|}{ Planting method }} & \multicolumn{3}{|c|}{$2002 / 2003$} & \multicolumn{3}{|c|}{$2003 / 2004$} \\
\hline & & GSS & Endeavor & Mean & GSS & Endeavor & Mean \\
\hline \multirow{3}{*}{ D. seed open } & & \multicolumn{6}{|c|}{ Non-reducing sugars (g/100 g fresh weight) } \\
\hline & Priming & 4.16 & 4.27 & 4.21 & 4.76 & 4.37 & 4.56 \\
\hline & $\begin{array}{l}\text { Check } \\
\text { Mean }\end{array}$ & 3.34 & 4.14 & 3.74 & 3.06 & 3.91 & 3.48 \\
\hline \multirow[t]{3}{*}{ D. seed Tunnel } & Priming & 4.48 & 3.94 & 4.21 & 5.33 & 3.64 & 4.48 \\
\hline & Check & 4.44 & 3.47 & 3.95 & 4.19 & 2.76 & 3.47 \\
\hline & Mean & 4.46 & 3.70 & 4.08 & 4.76 & 3.20 & 3.98 \\
\hline \multirow[t]{3}{*}{ Transplanting open } & Priming & 3.97 & 4.30 & 4.13 & 3.38 & 3.69 & 3.53 \\
\hline & Check & 3.46 & 3.98 & 3.72 & 2.93 & 3.07 & 3.00 \\
\hline & Mean & 3.71 & 4.14 & 3.92 & 3.15 & 3.38 & 3.26 \\
\hline \multirow[t]{6}{*}{ Transplanting tunnel } & Priming & 4.43 & 4.12 & 4.27 & 3.91 & 3.02 & 3.46 \\
\hline & Check & 3.89 & 3.24 & 3.56 & 3.09 & 2.59 & 2.84 \\
\hline & Mean & 4.16 & 3.68 & 3.92 & 3.50 & 2.80 & 3.15 \\
\hline & Priming & 4.26 & 4.16 & 4.21 & 4.14 & 3.68 & 3.91 \\
\hline & Check & 3.78 & 3.71 & 3.74 & 3.32 & 3.08 & 3.20 \\
\hline & Mean & 4.02 & 3.93 & & 3.73 & 3.38 & \\
\hline \multicolumn{2}{|c|}{ LSD at $5 \%$ level for Hybrid $(\mathrm{H})$} & \multicolumn{3}{|c|}{ NS } & \multicolumn{3}{|c|}{0.19} \\
\hline \multicolumn{2}{|c|}{ Planting method (PM) } & \multicolumn{3}{|c|}{ NS } & \multicolumn{3}{|c|}{0.27} \\
\hline \multicolumn{2}{|l|}{ Seed priming (SP) } & \multicolumn{3}{|c|}{0.29} & \multicolumn{3}{|c|}{0.19} \\
\hline $\mathrm{H} \times \mathrm{PM}$ & & \multicolumn{3}{|c|}{0.59} & \multicolumn{3}{|c|}{0.38} \\
\hline $\mathrm{H} \times \mathrm{SP}$ & & \multicolumn{3}{|c|}{ NS } & \multicolumn{3}{|c|}{0.26} \\
\hline $\mathrm{PM} \times \mathrm{SP}$ & & \multicolumn{3}{|c|}{ NS } & & NS & \\
\hline $\mathrm{H} \times \mathrm{PM} . \times \mathrm{SP}$ & & & NS & & & NS & \\
\hline & & & Reducing & $\operatorname{lgars}(\mathrm{g})$ & $0 \mathrm{~g}$ fres & eight) & \\
\hline D. seed open & Priming & 1.46 & 1.02 & 1.24 & 1.64 & 1.02 & 1.33 \\
\hline & Check & 1.69 & 0.95 & 1.32 & 1.89 & 0.89 & 1.39 \\
\hline & Mean & 1.57 & 0.98 & 1.28 & 1.76 & 0.95 & 1.36 \\
\hline D. seed Tunnel & Priming & 1.32 & 1.08 & 1.20 & 1.44 & 1.22 & 1.33 \\
\hline & Check & 1.26 & 1.25 & 1.25 & 1.18 & 1.45 & 1.31 \\
\hline & Mean & 1.29 & 1.16 & 1.22 & 1.31 & 1.33 & 1.32 \\
\hline Transplanting open & Priming & 1.28 & 0.94 & 1.11 & 1.51 & 1.11 & 1.31 \\
\hline & Check & 1.53 & 0.85 & 1.19 & 1.70 & 0.87 & 1.28 \\
\hline & Mean & 1.40 & 0.89 & 1.50 & 1.60 & 0.99 & 1.29 \\
\hline Transplanting tunnel & Priming & 1.10 & 0.98 & 1.04 & 1.47 & 1.19 & 1.33 \\
\hline & Check & 1.16 & 1.19 & 1.17 & 1.29 & 1.31 & 1.30 \\
\hline & Mean & 1.13 & 1.08 & 1.10 & 1.38 & 1.25 & 1.31 \\
\hline Mean & Priming & 1.29 & 1.01 & 1.15 & 1.51 & 1.13 & 1.32 \\
\hline & Check & 1.41 & 1.06 & 1.23 & 1.51 & 1.13 & 1.32 \\
\hline & Mean & 1.35 & 1.03 & & 1.51 & 1.13 & \\
\hline LSD at $5 \%$ level for & ybrid $(\mathrm{H})$ & & 0.18 & & & 0.18 & \\
\hline Planting method (PN) & & & NS & & & NS & \\
\hline Seed priming (SP) & & & NS & & & NS & \\
\hline $\mathrm{H} \times \mathrm{PM}$ & & & NS & & & 0.36 & \\
\hline $\mathrm{H} \times \mathrm{SP}$ & & & NS & & & NS & \\
\hline $\mathrm{PM} \times \mathrm{SP}$ & & & NS & & & NS & \\
\hline $\mathrm{H} \times \mathrm{PM} . \times \mathrm{SP}$ & & & NS & & & NS & \\
\hline
\end{tabular}


differences were noticed among planting methods, seed priming and all studied interactions on the reducing sugar content of ears in both seasons, with one exception, i.e., the interaction between hybrids and planting methods in the second season.

\section{Total sugars}

As presented in Table (6), kernels of GSS $9377 \mathrm{~F}_{1}$ hybrid contained higher total sugar percent compared to those of Endeavor $F_{1}$ hybrid in 2003 and 2004 seasons. Concerning planting method, direct seeding methods either in the open field or under tunnel gave higher total sugar percent compared to transplanting methods either in the open field or under tunnel. But the difference was not significant in the first season. Similarly, insignificant increase was observed for seed priming in 2003 season but seed priming increased significantly total sugars percent in 2004 season.

Concerning the interactions, no significant differences were recorded for all tested interaction except for hybrid combined with planting methods in the second season. GSS $9377 \mathrm{~F}_{1}$ hybrid with direct seeding under tunnel recorded the highest value of total sugar percent.

\section{Starch}

Data presented in Table (6) showed that there were no significant differences in starch values for any tested factor or their possible interactions. This result was true for both seasons.

\section{Dry matter}

There was no significant difference in dry matter percent of kernels between the studied hybrids in the first season, whereas Endeavor $F_{1}$ hybrid showed higher dry matter percent than GSS 9377 $F_{1}$ hybrid in the second season (Table, 7). Concerning planting methods, no significant difference was recorded among the planting methods in the first season while transplanting methods either in the open field or under tunnel recorded the highest value in dry matter in the second season. Regarding seed priming, the obtained data showed that seed priming increased dry matter percent in kernels compared with check treatment in both season.

Concerning the interactions, no significant differences were recorded for the investigated interactions of the three studied factors.

\section{Total soluble solids (TSS)}

Data in Table (7) indicated that GSS $9377 \mathrm{~F}_{1}$ hybrid kernels recorded higher values of TSS compared to those of Endeavor $F_{1}$ hybrid in both seasons. As for planting methods, no significant difference in TSS was found among the investigated direct seeding and transplanting methods in both seasons. The obtained data indicated also that seed priming increased the TSS values of kernels compared with check treatment in both seasons.

Concerning the studied interactions of the three tested factors, no significant differences were recorded in both seasons, except for the interaction between hybrid and planting methods in the second season. GSS $9377 \mathrm{~F}_{1}$ hybrid with transplanting under tunnel was the best treatment in increasing TSS in the second season.

\section{Yield}

\section{Early yield}

Plants of GSS $9377 \mathrm{~F}_{1}$ hybrid recorded higher early yield compared to Endeavor $F_{1}$ hybrid for both seasons. GSS $9377 \mathrm{~F}_{1}$ hybrid increased the early yield by $26.27 \%$ and $27.54 \%$ compared to Endeavor $F_{1}$ hybrid in 2003 and 2004 seasons, respectively (Table 8). Transplanting methods either in the open field or under tunnels gave higher early yield compared to direct seeding methods for both seasons. Similar results was reported by Miller (1972); Wyatt \& Mullins (1989); Vik \& Susort (1990); Waters et al (1990); Felczynki et al (1994) and Welbaum et al (2001), who found that transplanting gave higher early yield than direct seeding.

Concerning seed priming, seeds treated with PEG enhanced the early yield significantly in both seasons compared to the check treatment. Seed priming increased early yield by $26.91 \%$ and $24.58 \%$ in 2003 and 2004 seasons, respectively.

Regarding the interactions, plants of GSS 9377 $F_{1}$ hybrid transplanted under tunnel recorded the highest early yield compared to other treatments. The interaction among hybrid, planting method and seed priming showed that GSS $9377 \mathrm{~F}_{1}$ hybrid transplanted under tunnels using primed seeds produced significantly the highest early yield in both seasons.

\section{Total yield}

GSS $9377 \mathrm{~F}_{1}$ hybrid plants produced higher total yield than those of Endeavor $F_{1}$ hybrid as 
Table 6. Effect of hybrid, planting method and seed priming on total sugars $\%$ and starch $\%$ in kernels of sweet corn $F_{1}$ hybrids in 2002/2003 and 2003/2004 seasons

\begin{tabular}{|c|c|c|c|c|c|c|c|}
\hline \multirow{2}{*}{\multicolumn{2}{|c|}{ Planting method }} & \multicolumn{3}{|c|}{$2002 / 2003$} & \multicolumn{3}{|c|}{$2003 / 2004$} \\
\hline & & GSS & Endeavor & Mean & GSS & Endeavor & Mean \\
\hline \multirow{4}{*}{ D. seed open } & & \multicolumn{6}{|c|}{ Total sugars (g/100 g fresh weight) } \\
\hline & Priming & 5.62 & 5.29 & 5.45 & 6.40 & 5.39 & 5.89 \\
\hline & Check & 5.03 & 5.09 & 5.06 & 4.96 & 4.81 & 4.88 \\
\hline & Mean & 5.32 & 5.19 & 5.25 & 5.68 & 5.10 & 5.38 \\
\hline \multirow[t]{3}{*}{ D. seed Tunnel } & Priming & 5.79 & 5.02 & 5.40 & 6.77 & 4.88 & 5.82 \\
\hline & Check & 5.70 & 4.72 & 5.21 & 5.37 & 4.21 & 4.79 \\
\hline & Mean & 5.74 & 4.87 & 5.30 & 6.07 & 4.54 & 5.30 \\
\hline \multirow[t]{3}{*}{ Transplanting open } & Priming & 5.25 & 5.24 & 5.24 & 4.89 & 4.81 & 4.85 \\
\hline & Check & 4.99 & 4.84 & 4.91 & 4.64 & 3.93 & 4.28 \\
\hline & Mean & 5.12 & 5.04 & 5.07 & 4.76 & 4.37 & 4.56 \\
\hline \multirow[t]{3}{*}{ Transplanting tunnel } & Priming & 5.53 & 5.10 & 5.31 & 5.13 & 4.21 & 4.67 \\
\hline & Check & 5.05 & 4.43 & 4.74 & 4.38 & 3.90 & 4.14 \\
\hline & Mean & 5.29 & 4.76 & 5.02 & 4.75 & 4.05 & 4.40 \\
\hline \multirow[t]{3}{*}{ Mean } & Priming & 5.55 & 5.16 & 5.35 & 5.79 & 4.82 & 5.30 \\
\hline & Check & 5.19 & 4.77 & 4.98 & 4.84 & 4.21 & 4.52 \\
\hline & Mean & 5.37 & 4.97 & & 5.32 & 4.52 & \\
\hline \multicolumn{2}{|c|}{ LSD at 5\% level for Hybrid (H) } & & 0.40 & & & 0.29 & \\
\hline \multicolumn{2}{|c|}{ Planting method (PM) } & & NS & & & 0.41 & \\
\hline \multicolumn{2}{|l|}{ Seed priming (SP) } & & NS & & & 0.29 & \\
\hline \multicolumn{2}{|l|}{$\mathrm{H} \times \mathrm{PM}$} & & NS & & & 0.59 & \\
\hline \multicolumn{2}{|l|}{$\mathrm{H} \times \mathrm{SP}$} & & NS & & & NS & \\
\hline \multicolumn{2}{|l|}{$\mathrm{PM} \times \mathrm{SP}$} & & NS & & & NS & \\
\hline \multicolumn{2}{|l|}{$\mathrm{H} \times \mathrm{PM} . \times \mathrm{SP}$} & & NS & & & NS & \\
\hline \multirow{4}{*}{ D. seed open } & & \multicolumn{6}{|c|}{ Starch (g/100 g fresh weight) } \\
\hline & Priming & 1.84 & 1.44 & 1.64 & 1.80 & 1.71 & 1.75 \\
\hline & Check & 2.04 & 1.48 & 1.76 & 1.97 & 1.76 & 1.86 \\
\hline & Mean & 1.94 & 1.46 & 1.70 & 1.88 & 1.73 & 1.80 \\
\hline \multirow[t]{3}{*}{ D. seed Tunnel } & Priming & 1.35 & 1.75 & 1.55 & 1.50 & 1.81 & 1.65 \\
\hline & Check & 1.79 & 2.38 & 2.08 & 1.91 & 2.09 & 2.00 \\
\hline & Mean & 1.57 & 2.06 & 1.81 & 1.70 & 1.95 & 1.82 \\
\hline \multirow[t]{3}{*}{ Transplanting open } & Priming & 1.52 & 1.86 & 1.69 & 1.46 & 1.76 & 1.61 \\
\hline & Check & 1.68 & 2.65 & 2.16 & 1.97 & 2.06 & 2.01 \\
\hline & Mean & 1.60 & 2.25 & 1.92 & 1.71 & 1.91 & 1.81 \\
\hline \multirow[t]{3}{*}{ Transplanting tunnel } & Priming & 1.66 & 2.01 & 1.83 & 1.69 & 1.73 & 1.71 \\
\hline & Check & 2.03 & 2.06 & 2.04 & 1.89 & 2.10 & 1.99 \\
\hline & Mean & 1.84 & 2.03 & 1.93 & 1.79 & 1.91 & 1.85 \\
\hline Mean & Priming & 1.72 & 1.76 & 1.74 & 1.61 & 1.75 & 1.68 \\
\hline & Check & 1.88 & 2.14 & 2.01 & 1.93 & 2.00 & 1.96 \\
\hline & Mean & 1.80 & 1.95 & & 1.77 & 1.88 & \\
\hline LSD at $5 \%$ level for $\mathrm{Hy}$ & $\operatorname{rid}(\mathrm{H})$ & & NS & & & NS & \\
\hline Planting method (PM) & & & NS & & & NS & \\
\hline Seed priming (SP) & & & NS & & & NS & \\
\hline $\mathrm{H} \times \mathrm{PM}$ & & & NS & & & NS & \\
\hline $\mathrm{H} \times \mathrm{SP}$ & & & NS & & & NS & \\
\hline $\mathrm{PM} \times \mathrm{SP}$ & & & NS & & & NS & \\
\hline $\mathrm{H} \times$ PM. $\times \mathrm{SP}$ & & & NS & & & NS & \\
\hline
\end{tabular}


Table 7. Effect of hybrid, planting method and seed priming on dry matter \% and TSS in kernels of sweet corn $F_{1}$ hybrids in 2002/2003 and 2003/2004 seasons.

\begin{tabular}{|c|c|c|c|c|c|c|c|}
\hline \multirow{2}{*}{\multicolumn{2}{|c|}{ Planting method }} & \multicolumn{3}{|c|}{$2002 / 2003$} & \multicolumn{3}{|c|}{$2003 / 2004$} \\
\hline & & \multirow{3}{*}{$\begin{array}{r}\text { GSS } \\
20.49\end{array}$} & \multirow{3}{*}{$\begin{array}{l}\text { Endeavor } \\
16.35\end{array}$} & \multirow{2}{*}{\multicolumn{2}{|c|}{$\begin{array}{l}\text { Mean GSS } \\
\text { Dry matter \% }\end{array}$}} & \multirow{3}{*}{$\begin{array}{l}\text { Endeavor } \\
21.78\end{array}$} & \multirow{2}{*}{ Mean } \\
\hline & & & & & & & \\
\hline \multirow[t]{3}{*}{ D. seed open } & Priming & & & 18.42 & 23.34 & & 22.56 \\
\hline & Check & 19.84 & 16.18 & 18.01 & 17.21 & 21.65 & 19.43 \\
\hline & Mean & 20.16 & 16.26 & 18.21 & 20.27 & 21.71 & 20.99 \\
\hline \multirow{3}{*}{ D. seed Tunnel } & Priming & 17.79 & 18.25 & 18.02 & 21.53 & 20.46 & 20.99 \\
\hline & Check & 17.23 & 18.09 & 17.66 & 21.34 & 19.46 & 20.40 \\
\hline & Mean & 17.51 & 18.17 & 17.84 & 21.43 & 19.96 & 20.69 \\
\hline \multirow{3}{*}{ Transplanting open } & Priming & 20.46 & 16.29 & 18.37 & 21.34 & 24.81 & 23.07 \\
\hline & Check & 18.98 & 16.06 & 17.52 & 21.04 & 23.40 & 22.22 \\
\hline & Mean & 19.72 & 16.17 & 17.94 & 21.19 & 24.10 & 22.64 \\
\hline \multirow{3}{*}{ Transplanting tunnel } & Priming & 17.61 & 17.23 & 17.42 & 25.15 & 25.56 & 25.35 \\
\hline & Check & 17.56 & 17.20 & 17.38 & 20.11 & 23.85 & 21.98 \\
\hline & Mean & 17.85 & 17.21 & 17.40 & 22.63 & 24.70 & 23.66 \\
\hline \multirow[t]{3}{*}{ Mean } & Priming & 19.09 & 17.03 & 18.06 & 22.84 & 23.15 & 22.99 \\
\hline & Check & 18.40 & 16.88 & 17.64 & 19.92 & 22.09 & 21.01 \\
\hline & Mean & 18.74 & 16.96 & & 21.38 & 22.62 & \\
\hline \multicolumn{2}{|c|}{ LSD at 5\% level for Hybrid (H) } & & NS & & & 1.19 & \\
\hline \multicolumn{2}{|c|}{ Planting method (PM) } & & NS & & & 1.69 & \\
\hline \multirow{2}{*}{\multicolumn{2}{|c|}{$\begin{array}{l}\text { Seed priming (SP) } \\
\mathrm{H} \times \mathrm{PM}\end{array}$}} & & 0.89 & & & 1.19 & \\
\hline & & & NS & & & NS & \\
\hline \multicolumn{2}{|l|}{$\mathrm{H} \times \mathrm{SP}$} & & NS & & & NS & \\
\hline \multicolumn{2}{|l|}{$\mathrm{PM} \times \mathrm{SP}$} & & NS & & & NS & \\
\hline \multirow{2}{*}{\multicolumn{2}{|c|}{$\mathrm{H} \times \mathrm{PM} . \times \mathrm{SP}$}} & & NS & & & NS & \\
\hline & & \multicolumn{6}{|c|}{ Total soluble solids } \\
\hline \multirow{3}{*}{ D. seed open } & Priming & 16.07 & 13.15 & 14.61 & 15.50 & 13.99 & 14.74 \\
\hline & Check & 15.07 & 12.98 & 14.02 & 13.11 & 13.95 & 13.53 \\
\hline & Mean & 15.57 & 13.06 & 14.31 & 14.30 & 13.97 & 14.13 \\
\hline D. seed Tunnel & Priming & 16.04 & 13.25 & 14.64 & 15.01 & 14.29 & 14.65 \\
\hline & Check & 15.90 & 11.74 & 13.82 & 14.73 & 13.75 & 14.24 \\
\hline & Mean & 15.97 & 12.49 & 14.23 & $\mathbf{1 4 . 8 7}$ & 14.02 & 14.44 \\
\hline Transplanting open & Priming & 15.25 & 13.53 & 14.39 & 14.03 & 14.58 & 14.30 \\
\hline & Check & 14.67 & 12.56 & 13.61 & 13.56 & 14.13 & 13.84 \\
\hline & Mean & 14.96 & 13.04 & 14.00 & 13.79 & 14.35 & 14.07 \\
\hline Transplanting tunnel & Priming & 15.72 & 12.45 & 14.08 & 15.98 & 13.94 & 14.98 \\
\hline & Check & 15.52 & 12.00 & 13.76 & 14.89 & 13.63 & 14.26 \\
\hline & Mean & 15.62 & 12.22 & 13.92 & 15.43 & 13.78 & 14.61 \\
\hline Mean & Priming & 15.77 & 13.09 & 14.43 & 15.13 & 14.20 & 14.66 \\
\hline & Check & 15.29 & 12.32 & 13.80 & 14.07 & 13.86 & 13.96 \\
\hline & Mean & 15.53 & 12.71 & & 14.60 & 14.03 & \\
\hline LSD at $5 \%$ level for & Iybrid $(\mathrm{H})$ & & 0.49 & & & 0.53 & \\
\hline Planting method (P) & & & NS & & & NS & \\
\hline Seed priming (SP) & & & 0.49 & & & 0.53 & \\
\hline $\mathrm{H} \times \mathrm{PM}$ & & & NS & & & 1.63 & \\
\hline $\mathrm{H} \times \mathrm{SP}$ & & & NS & & & NS & \\
\hline $\mathrm{PM} \times \mathrm{SP}$ & & & NS & & & NS & \\
\hline $\mathrm{H} \times \mathrm{PM} . \times \mathrm{SP}$ & & & NS & & & NS & \\
\hline
\end{tabular}


Table 8. Effect of hybrid, planting method and seed priming on early yield of sweet corn $\mathrm{F}_{1}$ hybrids in 2002/2003 and 2003/2004 seasons.

\begin{tabular}{|c|c|c|c|c|c|c|c|}
\hline \multirow{2}{*}{\multicolumn{2}{|c|}{ Planting method }} & \multicolumn{3}{|c|}{$2002 / 2003$} & \multicolumn{3}{|c|}{$2003 / 2004$} \\
\hline & & GSS & Endeavor & Mean & GSS & Endeavor & Mean \\
\hline \multirow{4}{*}{ D. seed open } & & \multicolumn{6}{|c|}{ Early yield ton/feddan } \\
\hline & Priming & 8.34 & 8.54 & 8.44 & 7.61 & 6.89 & 7.25 \\
\hline & Check & 6.82 & 6.43 & 6.62 & 9.53 & 5.98 & 7.75 \\
\hline & Mean & 7.58 & 7.48 & 7.53 & 8.57 & 6.43 & 7.50 \\
\hline \multirow[t]{3}{*}{ D. seed Tunnel } & Priming & 10.77 & 6.57 & 8.67 & 8.89 & 6.38 & 7.63 \\
\hline & Check & 8.73 & 5.85 & 7.29 & 6.78 & 6.12 & 6.45 \\
\hline & Mean & 9.75 & 6.21 & 7.98 & 7.63 & 6.25 & 7.04 \\
\hline \multirow[t]{3}{*}{ Transplanting open } & Priming & 9.00 & 9.49 & 9.24 & 10.47 & 10.16 & 10.30 \\
\hline & Check & 7.18 & 6.60 & 6.89 & 8.61 & 6.65 & 7.63 \\
\hline & Mean & 8.09 & 8.04 & 8.06 & 9.54 & 8.40 & 8.97 \\
\hline \multirow[t]{3}{*}{ Transplanting tunnel } & Priming & 11.76 & 7.23 & 9.49 & 11.26 & 9.33 & 10.29 \\
\hline & Check & 8.90 & 5.94 & 7.42 & 10.24 & 6.03 & 8.13 \\
\hline & Mean & 10.33 & 6.58 & 8.45 & 10.75 & 7.68 & 9.21 \\
\hline \multirow[t]{3}{*}{ Mean } & Priming & 9.97 & 7.96 & 8.96 & 9.56 & 8.19 & 8.87 \\
\hline & Check & 7.91 & 6.21 & 7.06 & 8.04 & 6.19 & 7.12 \\
\hline & Mean & 8.94 & 7.08 & & 9.17 & 7.19 & \\
\hline \multicolumn{2}{|c|}{ LSD at 5\% level for Hybrid $(\mathrm{H})$} & & 0.33 & & & 0.34 & \\
\hline \multicolumn{2}{|c|}{ Planting method (PM) } & & 0.46 & & & 0.48 & \\
\hline \multicolumn{2}{|l|}{ Seed priming (SP) } & & 0.33 & & & 0.34 & \\
\hline \multicolumn{2}{|l|}{$\mathrm{H} \times \mathrm{PM}$} & & 0.66 & & & 0.67 & \\
\hline \multicolumn{2}{|l|}{$\mathrm{H} \times \mathrm{SP}$} & & NS & & & NS & \\
\hline \multicolumn{2}{|l|}{$\mathrm{PM} \times \mathrm{SP}$} & & NS & & & 0.67 & \\
\hline \multicolumn{2}{|l|}{$\mathrm{H} \times \mathrm{PM} . \times \mathrm{SP}$} & & 0.93 & & & 0.95 & \\
\hline \multirow{4}{*}{ D. seed open } & & \multicolumn{6}{|c|}{ Total yield (ton/feddan) } \\
\hline & Priming & 15.12 & 14.42 & 14.77 & 23.04 & 13.38 & 18.21 \\
\hline & Check & 13.79 & 12.88 & 13.33 & 22.32 & 11.27 & 16.79 \\
\hline & Mean & 14.45 & 13.65 & 14.05 & 22.68 & 12.32 & 17.50 \\
\hline \multirow{3}{*}{ D. seed Tunnel } & Priming & 18.62 & 13.79 & 16.20 & 23.67 & 11.50 & 17.58 \\
\hline & Check & 12.67 & 12.79 & 12.73 & 18.39 & 9.75 & 14.07 \\
\hline & Mean & 15.64 & 13.29 & 14.46 & 21.03 & 10.62 & 15.82 \\
\hline \multirow[t]{3}{*}{ Transplanting open } & Priming & 13.45 & 12.52 & 12.98 & 16.25 & 11.82 & 14.03 \\
\hline & Check & 9.61 & 9.44 & 9.52 & 14.41 & 11.30 & 12.85 \\
\hline & Mean & 11.53 & 10.98 & 11.25 & 15.33 & 11.56 & 13.44 \\
\hline \multirow[t]{3}{*}{ Transplanting tunnel } & Priming & 14.58 & 10.98 & 12.78 & 17.10 & 10.74 & 13.92 \\
\hline & Check & 10.27 & 9.04 & 9.65 & 15.72 & 9.83 & 12.77 \\
\hline & Mean & 12.42 & 10.01 & 11.21 & 16.41 & 10.28 & 13.34 \\
\hline Mean & Priming & 15.44 & 12.93 & 12.93 & 20.02 & 11.86 & 15.94 \\
\hline & Check & 11.58 & 11.04 & 11.04 & 17.71 & 10.54 & 14.12 \\
\hline & Mean & 13.51 & 11.98 & & 18.86 & 11.19 & \\
\hline LSD at $5 \%$ level for $\mathrm{Hy}$ & $\operatorname{rid}(\mathrm{H})$ & & 0.79 & & & 0.92 & \\
\hline Planting method (PM) & & & 1.12 & & & 1.30 & \\
\hline Seed priming (SP) & & & 0.79 & & & 0.92 & \\
\hline $\mathrm{H} \times \mathrm{PM}$ & & & 1.12 & & & NS & \\
\hline $\mathrm{H} \times \mathrm{SP}$ & & & NS & & & 1.84 & \\
\hline $\mathrm{PM} \times \mathrm{SP}$ & & & NS & & & NS & \\
\hline $\mathrm{H} \times \mathrm{PM} . \times \mathrm{SP}$ & & & NS & & & NS & \\
\hline
\end{tabular}


shown in Table (8), such increase amounted to $12.77 \%$ and $68.54 \%$ in the first and second seasons, respectively. Direct seeding either in the open field or under tunnels recorded higher total yield compared to transplanting methods in both seasons.

Similar results were reported by Vik \& Susort (1990) and Wyatt \& Akridge (1993). Treating seeds with PEG increased the total yield by $17.12 \%$ and $12.89 \%$ compared to those of check treatment in 2003 and 2004 seasons, respectively. Concerning the interactions, direct seeding plants of GSS $9377 \mathrm{~F}_{1}$ hybrids grown either in the open field or under tunnels recorded the higher total yield compared to those of transplanting, in both seasons. However, the difference was not significant in the second season. Seed priming of GSS $8377 \mathrm{~F}_{1}$ hybrids gave the highest total yield in both seasons compared to the other treatments of hybrids and seed priming interaction. The other interactions were not significant.

It could be concluded that the GSS $9377 \mathrm{~F}_{1}$ hybrid recorded the higher values in early and total yield. This might be due to increasing the plant growth parameters which might be reflected on the final performance in early and total yield or may be attributed to the genetic differences between the two hybrids.

Transplanting gave higher early yield compared to direct seeding. This might be attributed to that transplanting method permits more protection for plants during the cold weather at the early stage of plant growth. On the other hand, direct seeding methods increased the total yield compared to transplanting. This might be attributed to the stimulation of plant growth parameters due to direct seeding.

Also, seed priming increased the early yield and total yield through improvement of germination percent under stress condition and increasing the stand establishment, in addition to increasing the plant growth.

From this experiment we can conclude that regardless of the genetic differences in the two cultivars the effect of the planting methods coupled with seed priming by PEG treatments resulted in an economic impact per unit area in practical application.

In economic point of view about PEG treatments, it worth to mention that the Egyptian local price of $1 \mathrm{~kg}$ from PEG 6000 dalton equals $35 \mathrm{LE}$. (Egyptian pound), as we know one feddan take from 6-7 kg / grain seeds for planting, seed priming by use PEG 6000 dalton was $(400 \mathrm{~g} / \mathrm{L}$. of wa- ter) therefore, we can using about $1.6 \mathrm{~kg}$ PEG/ Fed., that coast 65 Egyptian pound to enhanced the early and total yield based on the results of the present study.

\section{REFERENCES}

Aguyoh, J.; H.G. Taber and V. Lawson (1999). Maturity of fresh market sweet corn with directseeded plants, transpla-nts, clear plastic mulch and row cover combinations. HorTechnology, 9(3): 420-425.

Bennett, M.A. (1986). Improving sweet corn germination and seedling growth at low temperature with seed hydration treatments. Dissertation Abstracts International, B. (Science and Engineering) 47 (1). 25 B Order No. DA 8606211. Field Crop Abstracts, 41. 8692.

Bennett, M.A. and Jr. L. Waters (1987a). Seed hydration treatments for improved sweet corn germination and stand establishment. J. Amer. Soc. Hort. Sci. 112 (1): 45-49.

Bennett, M.A. and Jr. L. Waters (1987b). Germination and emergence of high sugar sweet corn is improved by pre-sowing hydration of seed. HortScience, 22 (2): 236-238.

Bradford, K.J. (1986). Manipulation of seed water relations via osmotic priming to improve germination under stress conditions. HortScience, 21 (5): 1105-1112.

Felczynki, K.; I. Babik and J. Rumpel (1994). Plant and Soil covers in direct-seeded and transplanted sweet corn. Symp. on Timing of Field Production of Vegetables, Skierniewice, Poland, 23-27, 1993. (CAB Abstracts 1994-1996).

Miller, R.A. (1972). Forcing sweet corn. HortScience, 7(4): 424.

Nelson, N. (1974). A photometric adaptation of the Somogyi methods for determination of glucose. J. Biol. Chem. 195: 19-23.

Somogyi, M. (1952). Noted on sugar determination. J. Biol. Chem. 195: 19-23.

Snedecor, G.W. and W.G. Cochran (1989). Statistical Methods. 8 $^{\text {th }}$ Ed. Iowa State Univ. Press, Ames, Iowa, USA.

Vik, J. and A. Susort (1990). Cultivation trials in sweet corn. Norsk-landbruks-forsking 4(2): 107113. (CAB Abstracts 1992-1994).

Waters, Jr. L.; J.C. Alleyne; B. Shafii and S.K. Mohan (1990). Seed physiology, production \& technology combining vigor test results for prediction of final stand of shrunken-2 sweet corn seed. Crop Sci. 32: 1496-1505. 
Welbaum, G.E.; J.M. Frantz; M.K. Gunatilaka and $\mathrm{Z}$. Shen (2001). A comparison of the growth, establishment and maturity of direct-seeded and transplanted $\mathrm{Sh}_{2}$ sweet corn. HortScience 36 (4): 687-690.

Wyatt, J.E. and J.A. Mullins (1989). Production of sweet corn from transplants. HortScience, 24 (6): 1039.

Wyatt, J.E. and M.C. Akridge (1993). Yield and quality of direct-seeded and transplanted super sweet corn hybrids. Tennessee Farm and Home Science 167, 13-16. (CAB Abstracts1994).
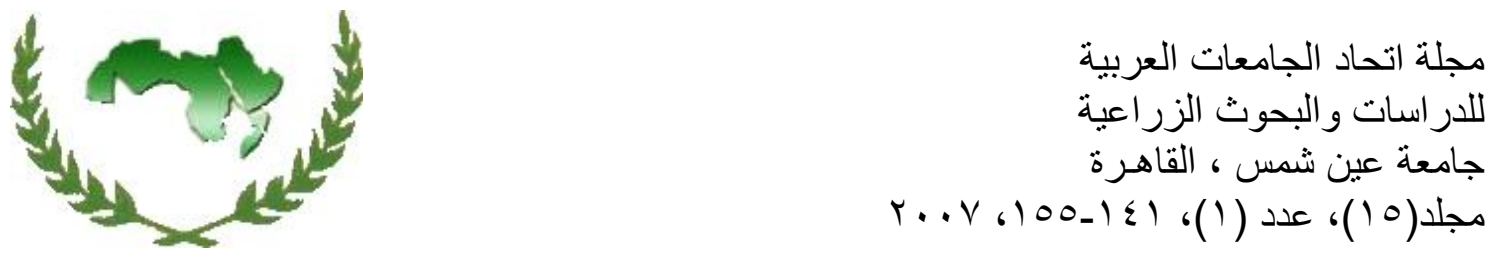

تأثير المعاملة بالبولي أيثيلين جليكول وطرق الزراعة علي إنتاجية الذرة السكرية

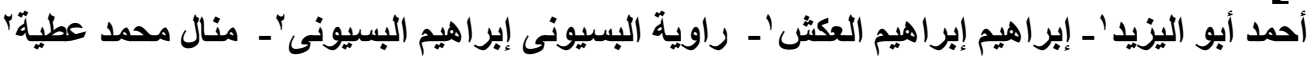

$\left[\begin{array}{lll}1 & \mathrm{r}\end{array}\right]$

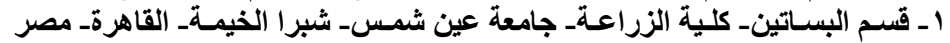

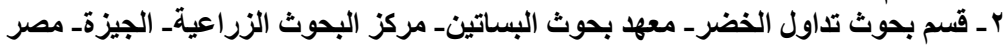

الغـلاف وكذللك المحصـول الكلي وذلك عند مقارنتهـا بطرق زر اعـة الـذرة السكــرية بالثـتلـ. الثــل أنـتج

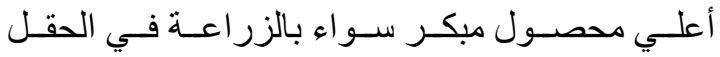

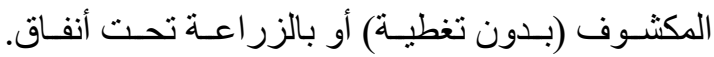

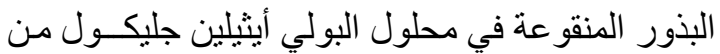

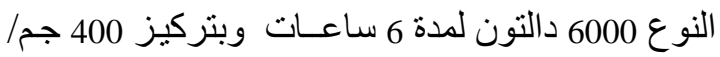
لتر أنتجبت أكثر النباتـات طو لاً مـع أكبر سمك لســــاق

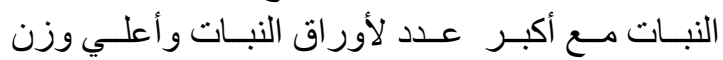

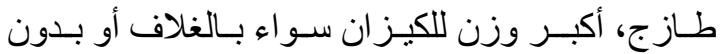

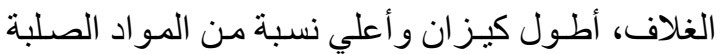

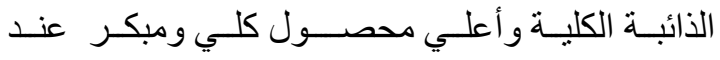
مقارنتها بالبذور غير المعاملة.
أجريت هذه الدراسة في تربة طميية بالمحطة التجرييية

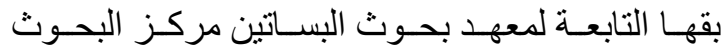

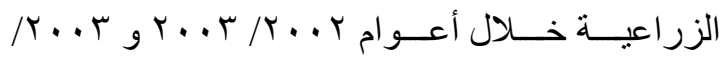

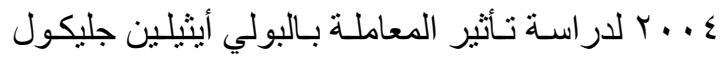

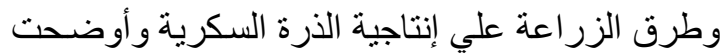

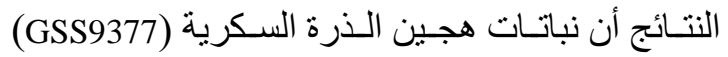
أعطت أفضل قوة في النمو و أعلي محصول مبكر وكلي

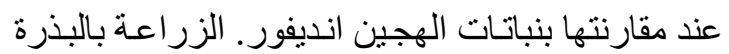

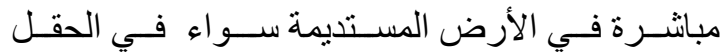

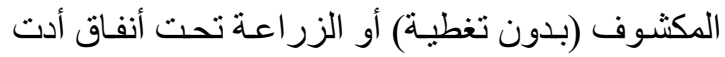

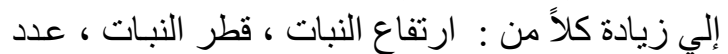

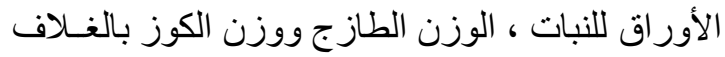

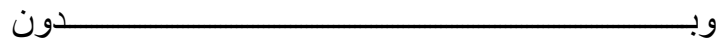

